\title{
The Mechanism of Plasma Plume Termination for Pulse Excited Plasmas in a Quartz Tube
}

Mingzhe Rong

Wenjie Xia

Xiaohua Wang

Zhijie Liu

Dingxin Liu

See next page for additional authors

Follow this and additional works at: https://digitalcommons.odu.edu/bioelectrics_pubs

Part of the Plasma and Beam Physics Commons

\section{Repository Citation}

Rong, Mingzhe; Xia, Wenjie; Wang, Xiaohua; Liu, Zhijie; Liu, Dingxin; Liang, Zhihu; Zhang, Xiaoning; and Kong, Michael G., "The Mechanism of Plasma Plume Termination for Pulse Excited Plasmas in a Quartz Tube" (2017). Bioelectrics Publications. 144.

https://digitalcommons.odu.edu/bioelectrics_pubs/144

\section{Original Publication Citation}

Mingzhe, R., Wenjie, X., Xiaohua, W., Zhijie, L., Dingxin, L., Zhihu, L., .. Kong, M. G. (2017). The mechanism of plasma plume termination for pulse-excited plasmas in a quartz tube. Applied Physics Letters, 111(7), 1-4. doi: 10.1063/1.4985332

This Article is brought to you for free and open access by the Frank Reidy Research Center for Bioelectrics at ODU Digital Commons. It has been accepted for inclusion in Bioelectrics Publications by an authorized administrator of ODU Digital Commons. For more information, please contact digitalcommons@odu.edu. 
Authors

Mingzhe Rong, Wenjie Xia, Xiaohua Wang, Zhijie Liu, Dingxin Liu, Zhihu Liang, Xiaoning Zhang, and Michael G. Kong 


\title{
The mechanism of plasma plume termination for pulse-excited plasmas in a quartz tube
}

\author{
Mingzhe Rong, ${ }^{1}$ Wenjie Xia, ${ }^{1}$ Xiaohua Wang, ${ }^{1, a)}$ Zhijie Liu, ${ }^{1}$ Dingxin Liu, ${ }^{1, a)}$ Zhihu Liang, ${ }^{2}$ \\ Xiaoning Zhang, ${ }^{2}$ and Michael G. Kong ${ }^{1,3,4}$ \\ ${ }^{1}$ State Key Laboratory of Electrical Insulation and Power Equipment, Center for Plasma Biomedicine, \\ Xi' an Jiaotong University, Xi' an 710049, People's Republic of China \\ ${ }^{2}$ Key Laboratory of Physical Electronics and Devices of the Ministry of Education, Xi' an Jiaotong University, \\ Xi' an 710049, People's Republic of China \\ ${ }^{3}$ Frank Reidy Center for Bioelectrics, Old Dominion University, Norfolk, Virginia 23508, USA \\ ${ }^{4}$ Department of Electrical and Computer Engineering, Old Dominion University, Norfolk, Virginia 23529, \\ USA
}

(Received 30 May 2017; accepted 9 August 2017; published online 17 August 2017)

\begin{abstract}
Although the formation and propagation of plasma plume for atmospheric pressure plasmas have been intensively studied, how does the plasma plume terminate is still little known. In this letter, helium plasma plumes are generated in a long quartz tube by pulsed voltages and a constant gas flow. The voltages have a variable pulse width (PW) from $0.5 \mu \mathrm{s}$ to $200 \mu \mathrm{s}$. It is found that the plasma plume terminates right after the falling edge of each voltage pulse when $\mathrm{PW}<20 \mu \mathrm{s}$, whereas it terminates before the falling edge. When PW is larger than $30 \mu \mathrm{s}$, the duration of plasma plume starts to decrease, and the termination is found to occur at the current zero moment of the discharge current through the high-voltage electrode, which is much different from that through the ground electrode. This indicates that part of the discharge current is shunted by the plasma plume to its downstream gas region. An equivalent circuit model is developed, from which the surface charge deposited on the quartz tube is found crucial for accelerating the termination of a plasma plume when PW > $30 \mu$ s. Published by AIP Publishing. [http://dx.doi.org/10.1063/1.4985332]
\end{abstract}

Atmospheric pressure plasmas have attracted growing attention thanks to their wide range of promising applications, such as plasma biomedicine ${ }^{1-4}$ and surface functionization. $^{5-7}$ Up to now, there have been many studies on the formation and propagation of plasma plumes in atmospheric pressure plasmas. ${ }^{8-10}$ For example, Mericam-Bourdet et al. predicted that the solitary surface ionization wave is crucial for the formation of a plasma plume, ${ }^{11}$ while Lu et al. proposed a photo-ionization theory to explain the plume's propagation. $^{12}$ Besides, Shao et al. pointed out that runaway electrons play an important role in high-speed propagation. ${ }^{13}$ However, so far the reports on the termination of plasma plumes are very few, and the underlying termination mechanism is not well understood. For example, it is generally thought that a plasma plume terminates right after the end of a voltage pulse due to the neutralization reactions in the ionized channel, ${ }^{14,15}$ implying that the length of plasma plume should increase with the pulse width. However, Lu et al. showed that an increase of the pulse width leads to a shorter plasma plume, ${ }^{16}$ which is contrary to the common notion. Further elucidating the termination mechanism of plasma plume is of scientific importance, since atmospheric pressure plasmas are a hot area of research, and technically beneficial for optimizing the plasma plume to meet application requirements, such as the application of a long plasma plume for root-canal disinfection. ${ }^{17}$

In this letter, a plasma source with a coaxial needle-toring electrode configuration was used. The needle electrode

\footnotetext{
${ }^{a)}$ Electronic addresses: xhw@mail.xjtu.edu.cn and liudingxin@mail.xjtu. edu.cn
}

was connected to a high-voltage pulsed power source, and the ring electrode was grounded [see Fig. 1(a)]. The needle tip was covered by hot glue to make the plasma plume more diffuse in a quartz tube, and the ring electrode was wrapped around the tube with a width of $2 \mathrm{~cm}$. The distance between the needle and the ring electrodes was $5 \mathrm{~mm}$ in the axial direction of the tube, and the tube had an inner diameter of $4 \mathrm{~mm}$, an outer diameter of $6 \mathrm{~mm}$, and a length of $70 \mathrm{~cm}$. Helium (5N) was used as the working gas, which flowed through the tube at a rate of $5 \mathrm{~L} / \mathrm{min}$. A homemade adjustable pulsed voltage generator was used, and the applied voltage had a flat-top value of $5 \mathrm{kV}$, a frequency of $4 \mathrm{kHz}$, and a pulse rise time of $30 \mathrm{~ns}$. The pulse width (full width half maximum) was varied from $0.5 \mu$ s to $200 \mu \mathrm{s}$. A voltage probe (Tektronix P6015A) was used to measure the applied voltage, and two current probes (Pearson 2877) were used to measure the currents through the high-voltage electrode and the ground electrode. Photographs of the plasma plumes were taken by a camera (Nikon D7000) with an exposure time of $2 \mathrm{~s}$, and when studying the time evolution of a plasma plume, images were taken by an intensified charged-couple device (PI-Max3) with an exposure time of $20 \mathrm{~ns}$.

The waveforms of the applied voltage are shown in Fig. 1(b), which almost overlap with each other except for their different pulse widths. This implies that any change of the plasma plume (will be discussed below) is caused by the pulse width, not by the other parameters of the applied voltage. Correspondingly, the waveforms of discharge current through the high-voltage electrode are also shown in Fig. 1(b). It can be seen that the current has a positive pulse at the rising edge of the voltage and a negative pulse at the falling 


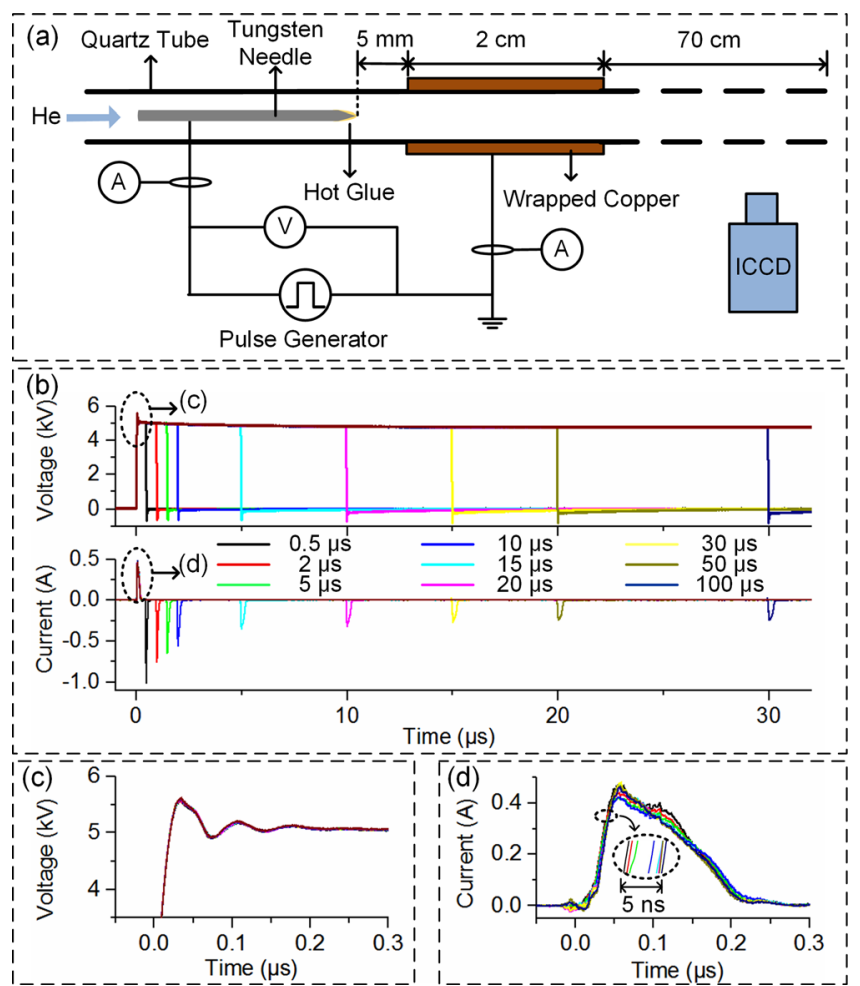

FIG. 1. (a) Schematic diagram of the experimental setup. (b) The voltage and current (through high-voltage electrode) waveforms for different pulse widths from 0.5 to $100 \mu \mathrm{s}$. (c) The rising edge waveforms of the voltage and (d) the corresponding current pulses.

edge of the voltage. This is similar to other reports of pulseexcited discharges as a result of the reverse breakdown. ${ }^{14,15}$ Interestingly, the negative current pulse becomes weaker and weaker with the increasing pulse width of the voltage from 0.5 to $20 \mu$ s, beyond that it is almost constant.

The rising edges of the voltage for different pulse widths are shown in Fig. 1(c), which further illustrate the good overlap of the voltage waveforms. However, the corresponding current waveforms do not overlap as shown in Fig. 1(d). They have similar pulse shape but different time lags to the discharge voltage. The dashed inset shows that the time lag increases with the pulse width of the voltage.

Figure 2(a) shows the photographs of the plasma plume for different pulse widths of the applied voltage. It can be seen that the length of plasma plume first increases, then decreases, and finally becomes almost invariable. Figure 2(b) shows the length of the plasma plume, its time duration, and the dissipated energy of one positive current pulse for pulse widths from 0.5 to $100 \mu \mathrm{s}$. It can be seen that the length of plasma plume increases almost linearly when $\mathrm{PW}<20 \mu \mathrm{s}$, and then, it gradually reaches a peak value of $\sim 43 \mathrm{~cm}$ at $\mathrm{PW} \sim 23 \mu$ s. After that, it decreases until $\mathrm{PW} \sim 50 \mu$ s to get into an almost constant value of $\sim 30 \mathrm{~cm}$. Similarly, the time duration of plasma plume also increases linearly until $\mathrm{PW}<20 \mu \mathrm{s}$, and in that case, it is slightly larger than the pulse width. After that, the time duration of the plasma plume becomes smaller than the pulse width, and it changes to decrease when PW $>30 \mu$ s. Note that for pulse widths above $50 \mu \mathrm{s}$, the length of plasma plume is almost constant, but the duration of the plasma plume decreases, which is attributed to the increase in propagation speed of the plasma plume as

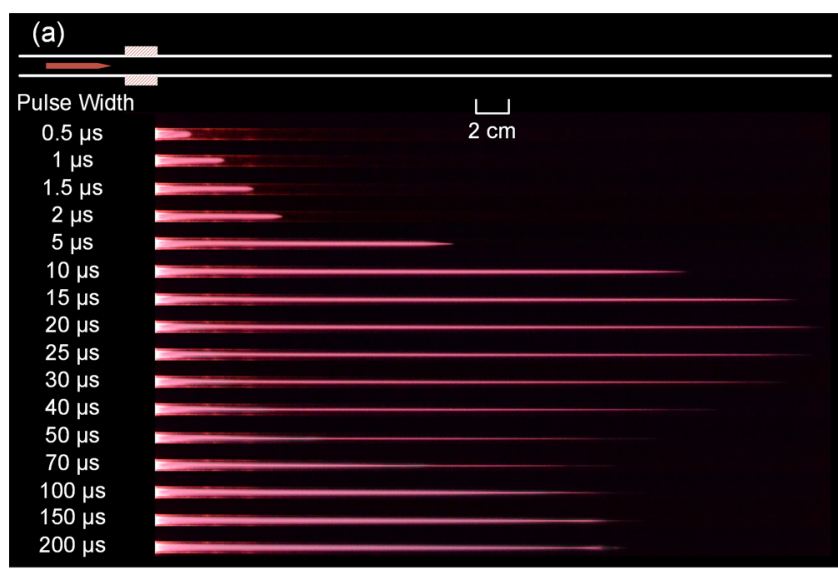

(b)

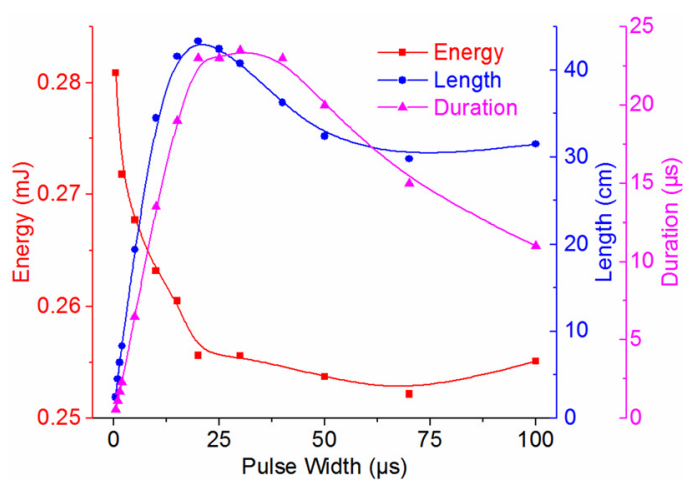

FIG. 2. (a) Photographs of the plasma plumes for different voltage pulse widths with an exposure time of $2 \mathrm{~s}$. (b) The curves of the plasma plume length, the duration of plasma plume, and the dissipated energy of one positive current pulse for pulse widths from 0.5 to $100 \mu \mathrm{s}$.

will be discussed below. As shown in Fig. 2(b), the dissipated energy of one positive current pulse, corresponding to the front edge of a voltage pulse, decreases from 0.28 to $0.25 \mathrm{~mJ}$ with the increasing pulse width from 0.5 to $20 \mu$ s, and then it maintains at $\sim 0.25 \mathrm{~mJ}$. This trend is similar to the peak value of the negative current pulse as presented above.

The time-resolved images of plasma plumes, i.e., the plasma bullets, are shown in Fig. 3(a) for the typical cases with $\mathrm{PW}=2,20$, and $100 \mu$ s. All the plasma bullets for a plasma plume are put together to form a mosaic photo, which accompanies the ordinary photo (taken by the Nikon camera) for comparison. The time interval for two adjacent bullets was kept at $250 \mathrm{~ns}$ by adjusting the trigger delay for the ICCD camera in each voltage cycle, and the exposure time was set at $20 \mathrm{~ns}$. The mosaic photo of the plasma plume has comparable length with the ordinary one when $\mathrm{PW}=2 \mu \mathrm{s}$, but it is shorter for $\mathrm{PW}=20$ or $100 \mu \mathrm{s}$ as a result of the two cameras' differences in exposure time and photosensitive efficiency. It can be seen that the plasma bullet becomes smaller and darker as it propagates and finally disappears. This is the case for $\mathrm{PW}=20$ and $100 \mu \mathrm{s}$, but for $\mathrm{PW}=2 \mu \mathrm{s}$, the bullet disappears suddenly when it is still big and bright. This implies that the plasma plume terminates due to the self-quenching reactions for the cases of $\mathrm{PW}=20$ and $100 \mu \mathrm{s}$, whereas it is forcibly terminated by the falling edge of the voltage pulse for $\mathrm{PW}=2 \mu \mathrm{s} .{ }^{11,14,15}$

Figure 3(b) shows that the initial velocity of the plasma bullet for $\mathrm{PW}=2 \mu \mathrm{s}$ is greater than that for the other two cases, which may be attributed to the larger dissipated energy at the front edge of the voltage pulse [see Fig. 2(b)]. 


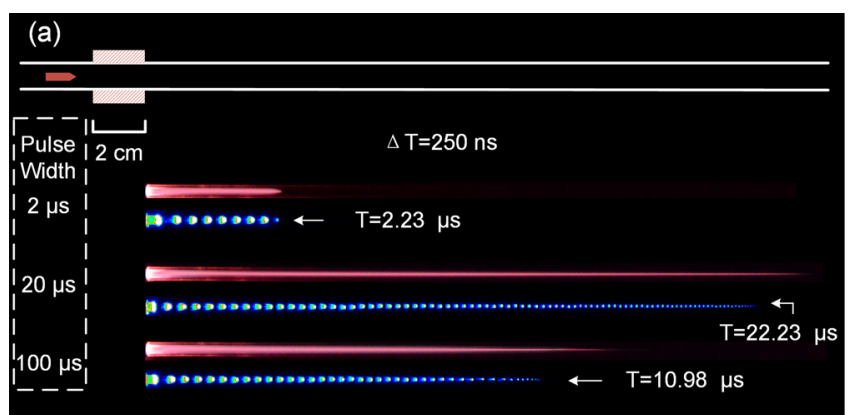

(b)

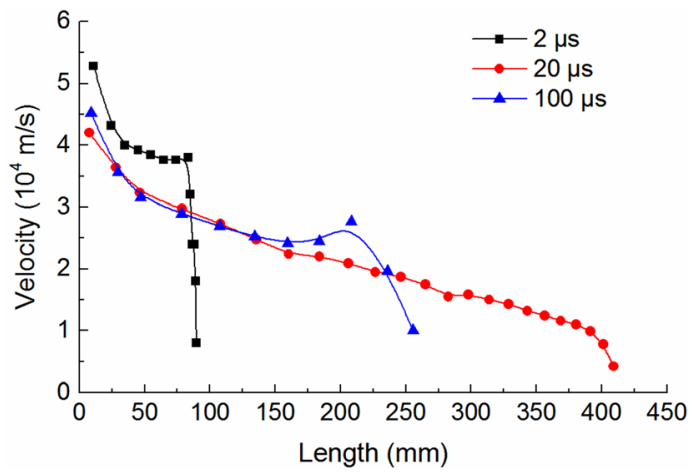

FIG. 3. (a) Time-resolved mosaic photographs of the plasma plumes and (b) velocity of the plasma bullets as a function of the plasma plume length for pulse widths of $2 \mu \mathrm{s}, 20 \mu \mathrm{s}$, and $100 \mu \mathrm{s}$.

However, the velocity suddenly drops with the falling edge of the voltage pulse at $2 \mu \mathrm{s}$, and the bullet disappears quickly within $0.23 \mu$ s. Similarly, the velocity of plasma bullet also drops quickly after the falling edge of the voltage pulse when PW $=20 \mu \mathrm{s}$, but in that case the velocity is very low of $1.2 \times 10^{4} \mathrm{~m} / \mathrm{s}$ just before the falling edge, and the plasma bullet is very small in that moment. This indicates that the plasma plume is about to terminate due to self-quenching reactions even without the falling edge of the voltage pulse. So, PW $\sim 20 \mu \mathrm{s}$ is a threshold value whether the selfquenching reaction or the falling edge of voltage pulse dominates the plasma plume termination. Interestingly, the plasma plume is the longest when $\mathrm{PW}=20 \mu$ s [see Fig. 2]. The plasma plume terminates at $\mathrm{t}=10.98 \mu \mathrm{s}$ when $\mathrm{PW}=100 \mu \mathrm{s}$, which is much before the falling edge of the voltage pulse.

Why does the plasma plume terminate faster with the increasing pulse width from $\mathrm{PW}=30 \mu$ s to $100 \mu$ s [see Fig. 2(b)]? It should not be directly caused by the falling edge of a voltage pulse because the termination moment is far ahead of that. In order to elucidate the underlying mechanism, we plotted in Fig. 4(a) the discharge currents either through the high-voltage electrode $\left(I_{\mathrm{hv}}\right)$ or through the ground electrode $\left(\mathrm{I}_{\mathrm{gr}}\right)$ for the pulse widths of $10,20,50$, and $100 \mu \mathrm{s}$. It can be seen that $\mathrm{I}_{\mathrm{gr}}$ decreases to zero at about $2 \mu$ s for all the cases of voltage pulses during their flat top region, but $\mathrm{I}_{\mathrm{hv}}$ lasts much longer. $\mathrm{I}_{\mathrm{hv}}$ is always larger than $\mathrm{I}_{\mathrm{gr}}$ when it is not zero. Therefore, $\mathrm{I}_{\mathrm{hv}}$ must be shunted by the plasma plume to its downstream gas region. In the scientific literature, the ground electrode current is commonly measured for plumecontaining plasmas such as a plasma jet, but here we note that the current through the high-voltage electrode is more important, because part of the current will not flow through the ground electrode. As shown in Fig. 4(a), $\mathrm{I}_{\mathrm{hv}}$ becomes

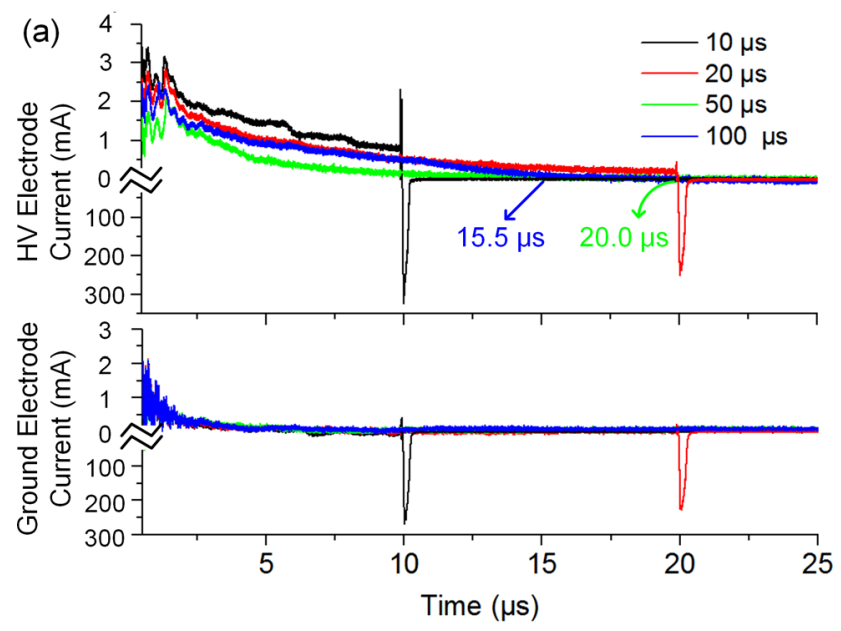

(b)

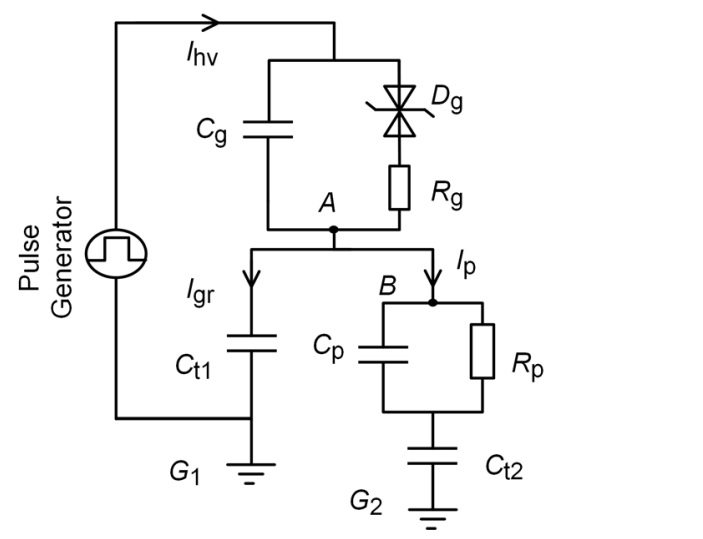

FIG. 4. (a) Discharge currents through the high-voltage electrode and ground electrode for $\mathrm{PW}=10,20,50$, and $100 \mu$ s. The waveforms are shown from 0.5 to $25 \mu \mathrm{s}$. (b) An equivalent circuit model for pulsed-excited plasmas in a quartz tube.

zero at the very moment of the falling edge for $\mathrm{PW}=10 \mu \mathrm{s}$ and $20 \mu$ s, but it decreases gradually to zero before the falling edge when $\mathrm{PW}>23 \mu \mathrm{s}$. For example, the current zero moment of $\mathrm{I}_{\mathrm{hv}}$ is $15.5 \mu \mathrm{s}$ for the case of $\mathrm{PW}=100 \mu \mathrm{s}$. Importantly, it is found that the current zero moments of $\mathrm{I}_{\mathrm{hv}}$ are almost the same as the moments of plasma plume termination when $\mathrm{PW}>30 \mu \mathrm{s}$. This is because the plasma plume needs electrical energy to be sustained.

In order to better elucidate the mechanism of plasma plume termination, an equivalent circuit model was proposed, as shown in Fig. 4(b). This model is based on a previous plasma jet model proposed by Lu et al. ${ }^{18}$ and a dielectric barrier discharge model proposed by Underwood et al. ${ }^{19}$ The equivalent circuit is composed of a circuit for the gas gap between the high-voltage and ground electrodes, which is in series with two current branches either to the ground electrode or to the downstream gas region. The two branches are parallel with each other. For the gas gap, $C_{g}$ is used to describe the gap capacitance, and a bidirectional zener diode $\mathrm{D}_{\mathrm{g}}$ and a variable resistance $\mathrm{R}_{\mathrm{g}}$ are used for the nonlinear gap resistance. The constant gap breakdown voltage can be represented by $\mathrm{D}_{\mathrm{g}}$, and the change in gas conductivity due to the space charge can be reflected by $\mathrm{R}_{\mathrm{g}}$. There is a quartz tube barrier between the gas gap and the ground electrode $\left(\mathrm{G}_{1}\right)$, and $\mathrm{C}_{\mathrm{t} 1}$ represents its capacitance. The current branch through the plasma plume to the downstream gas region consists of a capacitance $\left(C_{p}\right)$ and a resistance $\left(R_{p}\right)$ to reflect the 
ionized channel of the plasma plume. $\mathrm{C}_{\mathrm{t} 2}$ represents the barrier capacitance in the downstream gas region which is connected to the remote virtual ground $\left(\mathrm{G}_{2}\right)$.

For the equivalent circuit described in Fig. 4(b), the surface charge deposited on the quartz tube (represented by point $\mathrm{A}$ ) and the space charge in the gas gap play an important role in the plasma plume termination when $\mathrm{PW}<20 \mu$ s. The surface charge is produced by each discharge during the rising edge of the voltage pulse, and it is mainly diminished by the reverse discharge during the falling edge. Both the discharges at the rising and falling edges of a voltage pulse produce space charges, which are mainly diminished by neutralization reactions. After each discharge, the amount of space charge decreases sharply with time, leading to an increase of $\mathrm{R}_{\mathrm{g}}$ in the equivalent circuit. Therefore, it can be deduced that the current of the reverse discharge weakens with the increasing pulse width of the applied voltage, which agrees well with the experimental results shown Fig. 1(b). More surface charge will reside with the increasing $R_{g}$, and as a result, higher voltage is needed for the gas breakdown at the front edge of the next voltage pulse, which is verified by the increasing time lag of the discharge current [see Fig. 1(d)] and the decreasing discharge energy as shown in Fig. 2(b). The current for the reverse discharge becomes almost constant when PW $>20 \mu$ s, which may be because the residual time for the space charge is less than $20 \mu \mathrm{s}$. When $\mathrm{PW}<20 \mu$ s, the reverse discharge will not only diminish the surface charge but also terminate the plasma plume by the reverse electric field (the direction of $\mathrm{I}_{\mathrm{p}}$ reverses).

When PW $>30 \mu \mathrm{s}$, the plasma plume terminates before the falling edge of voltage pulse, and therefore, the reverse discharge will not influence the plasma termination directly. However, increasing pulse width leads to more surface charge driven by the electric field to the downstream region, and as a result, as a result more surface charge will reside on there [corresponding to the point B in Fig. 4(b)] when the next voltage pulse comes. This would hinder the propagation of the plasma plume, leading to decreasing length of plasma plume with the increasing pulse width when $30<\mathrm{PW}<50 \mu$ s (see Fig. 2). When $\mathrm{PW}>50 \mu$ s, the reverse discharge at the falling edge will enhance the discharge at the front edge of the next pulse since the time gap is less than $200 \mu \mathrm{s}$, so the length of the plasma plume stops decreasing. The enhancement can be reflected by the larger discharge current through the high-voltage electrode as shown in Fig. 4(a). However, the current for $\mathrm{PW}=100 \mu \mathrm{s}$ still decreases to zero faster than for $\mathrm{PW}=50 \mu \mathrm{s}$, because the surface charge is mainly on the downstream region, and consequently its effect is pronounced only when the plasma plume propagates long enough.

The length of the plasma plume is the longest when the pulse width is between 20 and $30 \mu$ s. This might be because the reverse discharge enhances the plasma plume when it is about to disappear via self-quenching reactions.

In summary, the purpose of this letter is to elucidate the termination mechanism of plasma plume for helium plasmas at atmospheric pressure. The plasma plumes were generated in a long quartz tube by flat-topped pulsed voltages and a constant gas flow. The voltages have a variable pulse width (PW) from 0.5 to $100 \mu \mathrm{s}$. It was found that when PW $<20 \mu$ s, the plasma plume terminates with the falling edge of a voltage pulse due to the reverse electric field formed in the plasma plume, whereas it terminates before the falling edge. When PW $>30 \mu$ s, the termination occurs when the discharge current through the high-voltage electrode decreases to zero, which is much different to the current through the ground electrode. This indicates that part of the discharge current is shunted by the plasma plume to its downstream region, which acts as a third part electrode. Based on this, an equivalent circuit model was developed. It was deduced that the surface charge on the quartz tube deposited by the last voltage pulse would inhibit the current one, and the amount of surface charge becomes greater with increasing pulse width, leading to an acceleration of plasma plume termination when PW $>30 \mu$ s. It should be noted that the termination mechanism of the plasma plume presented here is confined in a quartz tube, which might not be exactly the same as that for the helium plasma jets in open air, so further studies on the termination mechanism are still needed.

This work was supported by the National Natural Science Foundation of China (Grant No. 51521065). The authors would like to thank Dr. Han $\mathrm{Xu}$ for fruitful discussions related to this work.

${ }^{1}$ T. von Woedtke, S. Reuter, K. Masur, and K.-D. Weltmann, Phys. Rep. 530, 291 (2013).

${ }^{2}$ K. Lee, K. H. Paek, W. T. Ju, and Y. Lee, J. Microbiol. 44, 269 (2006).

${ }^{3}$ G. Fridman, G. Friedman, A. Gutsol, A. B. Shekhter, V. N. Vasilets, and A. Fridmanx, Plasma Processes Polym. 5, 503 (2008).

${ }^{4}$ M. G. Kong, G. Kroesen, G. Morfill, T. Nosenko, T. Shimizu, J. van Dijk, and J. L. Zimmermann, New J. Phys.11, 115012 (2009).

${ }^{5}$ J. L. Walsh and M. G. Kong, Appl. Phys. Lett. 91, 251504 (2007).

${ }^{6}$ Z. Fang, J. Lin, X. Xie, Y. Qiu, and E. Kuffel, J. Phys. D: Appl. Phys. 42, 085203 (2009).

${ }^{7}$ J. Xu, C. Zhang, T. Shao, Z. Fang, and P. Yan, J. Electrost. 71, 435 (2013).

${ }^{8}$ J. P. Boeuf, L. L. Yang, and L. C. Pitchford, J. Phys. D: Appl. Phys. 46, 015201 (2012).

${ }^{9}$ X. Lu, G. V. Naidis, M. Laroussi, and K. Ostrikov, Phys. Rep. 540, 123 (2014).

${ }^{10}$ X. Lu, G. V. Naidis, M. Laroussi, S. Reuter, D. B. Graves, and K. Ostrikov, Phys. Rep. 630, 1 (2016).

${ }^{11}$ N. Mericam-Bourdet, M. Laroussi, A. Begum, and E. Karakas, J. Phys. D: Appl. Phys. 42, 055207 (2009).

${ }^{12}$ X. Lu and M. Laroussi, J. Appl. Phys. 100, 063302 (2006).

${ }^{13}$ T. Shao, V. F. Tarasenko, C. Zhang, D. V. Rybka, I. D. Kostyrya, A. V. Kozyrev, P. Yan, and V. Y. Kozhevnikov, New J. Phys. 13, 113035 (2011).

${ }^{14}$ E. Karakas and M. Laroussi, J. Appl. Phys. 108, 063305 (2010).

${ }^{15}$ E. Karakas, M. A. Akman, and M. Laroussi, Plasma Sources Sci. Technol. 21, 034016 (2012).

${ }^{16}$ X. Lu, Z. H. Jiang, Q. Xiong, Z. Y. Tang, Z. L. Xiong, J. Hu, X. W. Hu, and Y. Pan, IEEE Trans. Plasma Sci. 36, 988 (2008).

${ }^{17}$ X. Lu, Y. Cao, P. Yang, Q. Xiong, Z. Xiong, Y. Xian, and Y. Pan, IEEE Trans. Plasma Sci. 37, 668 (2009).

${ }^{18}$ X. Lu, Z. H. Jiang, Q. Xiong, Z. Y. Tang, X. W. Hu, and Y. Pan, Appl. Phys. Lett. 92, 081502 (2008).

${ }^{19}$ T. Underwood, S. Roy, and B. Glaz, J. Appl. Phys 113, 083301 (2013). 